\title{
The Mixed Berge Equilibrium in Extensive Form Games
}

\author{
Ahmad Nahhas", H. W. Corley \\ Center on Stochastic Modeling, Optimization, and Statistics, The University of Texas at Arlington, Arlington, TX, USA \\ Email:ahmad.nahhas@mavs.uta.edu,corley@uta.edu
}

How to cite this paper: Nahhas, A. and Corley, H.W. (2017) The Mixed Berge Equilibrium in Extensive Form Games. Theoretical Economics Letters, 7, 2230-2238. https://doi.org/10.4236/tel.2017.77152

Received: November 4, 2017

Accepted: December 15, 2017

Published: December 18, 2017

Copyright $\odot 2017$ by authors and Scientific Research Publishing Inc. This work is licensed under the Creative Commons Attribution International License (CC BY 4.0).

http://creativecommons.org/licenses/by/4.0/

\section{cc) (i) Open Access}

\begin{abstract}
In this paper we apply the concept of a mixed Berge equilibrium to finite $n$-person games in extensive form. We study the mixed Berge equilibrium in both perfect and imperfect information finite games. In addition, we define the notion of a subgame perfect mixed Berge equilibrium and show that for a 2-person game, there always exists a subgame perfect Berge equilibrium. Thus there exists a mixed Berge equilibrium for any 2-person game in extensive form. For games with 3 or more players, however, a mixed Berge equilibrium and a subgame perfect mixed Berge equilibrium may not exist. In summary, this paper extends extensive form games to include players acting altruistically.
\end{abstract}

\section{Keywords}

Mixed Berge Equilibrium, Extensive Form Games, Game Theory

\section{Introduction}

The Berge equilibrium (BE) is a solution concept in game theory introduced in [1] and formally defined in [2]. It was extended to mixed strategies (MBE) in [3]. The Berge equilibrium represents a strategy that is mutually cooperative. In other words, at a Berge equilibrium player $i$ cannot gain a better payoff if any other player changes his strategy unilaterally. In effect, an MBE represents the situation where every $n-1$ players choose the best joint mixed strategy for the remaining player. In this paper, we apply the concept of an $\mathrm{MBE}$ to finite extensive form games, where players make decisions sequentially. We consider here finite $n$-person extensive form games both with complete information and incomplete information. In a complete information game, each player is aware of the actions \#Both authors contributed equally to this research. 
of the other players. In imperfect information games, however, players are not aware of the actions that other players choose.

The paper is organized as follows. In Section 2, we give the needed notation and definitions. In Section 3, we study the existence of an MBE in extensive form games. In Section 4, we give examples, and then give conclusions in Section 5.

\section{Preliminaries}

We use here a notation similar to that of [4]. An extensive form game $G$ is written as $G=(N, H, P, I)$, where $N$ is the set of the players, $H$ is the set of histories, $P$ is a function assigning a player to each non-terminal history, and $I$ represents an information set.

Each history $h$ is a sequence of actions $\left(a^{k}\right)_{k=1, \cdots, K}$. In this paper, we assume that all the sequences of actions are finite. Hence the game is finite. Each history $h \in H$ ends with a terminal node which gives the utility value for each of the $n$-players. Each non-terminal node belongs to an information set $I_{i}$ for a player $i$ such that $P(h)=i$. The set of all information sets for player $i$ is $\tau_{i}$. If each information set has only one node, then the game is a perfect information game. In an imperfect information game, two or more nodes belong to some information set. If two or more nodes belong to the same information set, then they are connected with a dotted line. The idea is that if an information set includes only one decision node, then a player knows the actions that led to that node so the game is a perfect information game. An example of a 2-person extensive form game is show in Figure 1. In this game, player 1 makes a decision $s_{1}$ or $t_{1}$. Next, player 2 makes a decision. After that, either the game is finished or player 1 makes a decision with imperfect information. The label above a node $(i: j)$ means information set $j$ for player $i$.

Each game in extensive form can be represented as a game in normal form [5]. The set of pure strategies $S_{i}=\left\{\times A_{i} \mid A_{i} \in I_{i}, I_{i} \in \tau_{i}\right\}$ for each player $i$ is the Cartesian product over the actions player $i$ has at each of his information sets. A mixed strategy $\sigma_{i}$ for a player $i$ is a probability distribution over his set of pure strategies. The set of all mixed strategies for player $i$ is $\Delta S_{i}$. The support of a

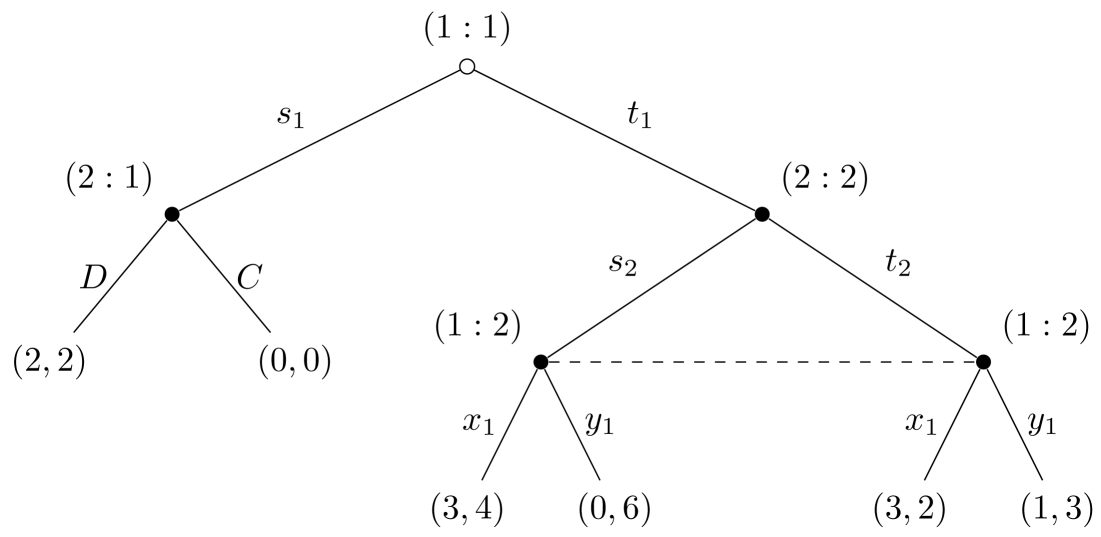

Figure 1. Example of a two-person extensive form game. 
mixed strategy for player $i$ is $\operatorname{supp}\left(\sigma_{i}\right)=\left\{s_{i} \in S_{i} \mid \sigma_{i}\left(s_{i}\right)>0\right\} \quad$ A pure strategy for player $i$ is a special case of the mixed strategy where a player chooses exactly one action at each of his information sets.

Similarly, a mixed strategy for all players other than player $i$ is a probability distribution $\sigma_{-i}$ over the set of the Cartesian product of all the pure strategies for all players other than player $i$. Hence $\sum_{s_{-i \in S_{-}}} \sigma_{-i}\left(s_{-i}\right)=1, \sigma_{-i}\left(s_{-i}\right) \geq 0$, where $\sigma_{-i}\left(s_{-i}\right)$ is the that product of the probabilities that each player other than player $i$ chooses the strategy $s_{-i}$. The set of all mixed strategies for all players other than player $i$ is $\Delta S_{-i}$. The support of a mixed strategy for all players other than player $i$ is $\operatorname{supp}\left(\sigma_{-i}\right)=\left\{s_{-i} \in S_{-i} \mid \sigma_{-i}\left(s_{-i}\right)>0\right\}$.

The following identities were derived in [3]. Player $i$ 's expected payoff for strategy $s_{i}$ for player $i$ and strategy $\sigma_{-i}$ for the remaining players is

$$
u_{i}\left(s_{i}, \sigma_{-i}\right)=\sum_{s_{-i} \in S_{-i}} \sigma_{-i}\left(s_{-i}\right) u_{i}\left(s_{i}, s_{-i}\right) .
$$

Player $i$ 's expected payoff for strategy $\sigma_{i}$ for player $i$ and strategy $s_{-i}$ for the remaining players is

$$
u_{i}\left(\sigma_{i}, s_{-i}\right)=\sum_{s_{i} \in S_{i}} \sigma_{i}\left(s_{i}\right) u_{i}\left(s_{i}, s_{-i}\right) .
$$

Player $i$ 's expected payoff for strategy $\sigma_{i}$ for player $i$ and strategy $\sigma_{-i}$ for the remaining players is

$$
u_{i}\left(\sigma_{i}, \sigma_{-i}\right)=\sum_{s_{i} \in S_{i} s_{-i} \in S_{-i}} \sigma_{i}\left(s_{i}\right) \sigma_{-i}\left(s_{-i}\right) u_{i}\left(s_{i}, s_{-i}\right) .
$$

We now define the NE.

Definition 1. A strategy $\sigma^{*}$ is an NE if and only if

$$
\max _{s_{i} \in S_{i}} u_{i}\left(s_{i}, \sigma_{i}^{*}\right)=u_{i}\left(\sigma_{i}^{*}, \sigma_{-i}^{*}\right) \geq u_{i}\left(\sigma_{i}, \sigma_{-i}^{*}\right), \forall \sigma_{i} \in \Delta S_{i}, \forall i \in N .
$$

In an NE, no player can increase his expected payoff by changing his strategy unilaterally. We can similarly define an MBE.

Definition 2. A strategy $\sigma^{*}$ is an MBE if and only if

$$
\max _{s_{-i} \in S_{-i}} u_{i}\left(\sigma_{i}^{*}, s_{-i}\right)=u_{i}\left(\sigma_{i}^{*}, \sigma_{-i}^{*}\right) \geq u_{i}\left(\sigma_{i}^{*}, \sigma_{-i}\right), \forall \sigma_{-i} \in \Delta S_{-i}, \forall i \in N .
$$

In an MBE all players other than player $i$ cannot increase the expected payoff for player $i$ by changing their strategies. Hence no player can increase other player's expected payoff by changing his strategy unilaterally.

Next, the subgame perfect Nash equilibrium (SPNE) is an important concept in extensive games since it always exists. An SPNE can be obtained using backward induction. The following definition of a subgame is from [4].

Definition 3. An extensive form subgame is a sequence of actions $h^{\prime}$ after a history $h$ such that $\left(h, h^{\prime}\right) \in H$.

We extend the concept of the SPNE to a subgame perfect MBE (SPMBE). We prove that one exists for every 2-person game. However, we show that one may not exist for $n \geq 3$.

Definition 4. A strategy $\sigma^{*}$ is an SPNE if and only if for every nonterminal history $h$ with $P(h)=i$, then 


$$
u_{i}\left(\left.\sigma_{i}^{*}\right|_{h},\left.\sigma_{-i}^{*}\right|_{h}\right) \geq u_{i}\left(\sigma_{i},\left.\sigma_{-i}^{*}\right|_{h}\right), \forall \sigma_{i} \in \Delta S_{i}, \forall i \in N
$$

An SPNE, is an NE for some subgame. Furthermore, no player can increase his expected payoff by changing his strategy unilaterally at any information node and history $h$ such that $P(h)=i$.

We now give the definition of an SPMBE. Note the difference in history as opposed to Definition 4.

Definition 5. A strategy $\sigma^{*}$ is an SPMBE if and only if for every non-terminal history $h$ with $P(h) \neq i$, then

$$
u_{i}\left(\left.\sigma_{i}^{*}\right|_{h},\left.\sigma_{-i}^{*}\right|_{h}\right) \geq u_{i}\left(\left.\sigma_{i}^{*}\right|_{h}, \sigma_{-i}\right), \forall h \in H, P(h) \neq i, \forall \sigma_{-i} \in \Delta S_{-i}, \forall i \in N .
$$

Thus an SPMBE is a subgame concept where a strategy is an MBE for some subgame. Furthermore, players other than player $i$ cannot increase player $i$ 's expected payoff by unilaterally changing their strategies at any information node with a non-terminal history $h$ for which $P(h) \neq i$.

\section{MBE Existence in Extensive Form Games}

We now consider the existence of an MBE in an extensive form game. The following theorem from [5] is used.

Theorem 1. Every game in extensive form has a subgame perfect NE.

Next we define a 2-person game $G^{\prime}$ in extensive form. Each player has the same set of actions as he has in the game $G$. However, the two players payoffs are swapped.

Definition 6. The game $G^{\prime}$ is a 2-person game where each player has the same actions as in the game $\mathrm{G}$. The payoffs for player 1 in $\mathrm{G}$ are the payoffs for player 2 in $G^{\prime}$ and vice versa.

Lemma 2. Let $\mathrm{G}$ be a 2-person normal form game. Then any NE for the game $G^{\prime}$ is an MBE for G.

Proof. Let $\sigma^{*}$ be an NE for $G^{\prime}$. Then,

$$
u_{1}\left(\sigma_{1}^{*}, \sigma_{2}^{*}\right) \geq u_{1}\left(\sigma_{1}^{*}, \sigma_{2}\right), \forall \sigma_{2} \in \Delta S_{2},
$$

and

$$
u_{1}\left(\sigma_{1}^{*}, \sigma_{2}^{*}\right) \geq u_{1}\left(\sigma_{1}^{*}, \sigma_{2}\right), \forall \sigma_{2} \in \Delta S_{2} .
$$

Thus $\sigma^{*}$ is an MBE by Definition 5 to complete the proof.

The following remark follows immediately from Theorem 1 and Lemma 2.

Remark. Every 2-person game G has an SPMBE. Hence every 2-person game in extensive form has an MBE.

Proof. Let $G$ be a 2-person game and $G^{\prime}$ is the game with the swapped payoffs for the two players. By Theorem 1, $G^{\prime}$ always has an SPNE $\sigma^{*}$ for some subgame in $G^{\prime}$. Therefore by Lemma 2, $\sigma^{*}$ is an MBE for the same subgame in $G$. Hence the game $G$ has an SPMBE. Moreover, by Definition 5 an SPMBE is an MBE for the game $G$, and the proof is complete.

In the following lemma, we give necessary and sufficient conditions for the 
existence on an MBE.

Lemma 3. A strategy $\sigma^{*}$ is an MBE for G if and only if $\sigma_{-i}^{*}\left(s_{-i}\right)=0$ when

$$
u_{i}\left(\sigma_{i}^{*}, s_{-i}\right)<\max _{s_{-i} \in S_{-i}} u_{i}\left(\sigma_{i}^{*}, s_{-i}\right) \text {. }
$$

Proof. Let $\sigma^{*}$ be an MBE for $G$. Suppose that there exists a strategy $S_{-i}$ such that $u_{i}\left(\sigma_{i}^{*}, s_{-i}\right)<\max _{s_{-i} \in S_{-i}} u_{i}\left(\sigma_{i}^{*}, s_{-i}\right)$ and $\sigma_{-i}\left(s_{-i}\right)>0$. Hence by Equation (3) $u_{i}\left(\sigma_{i}^{*}, \sigma_{-i}^{*}\right)<\max _{s_{-i} \in S_{-i}} u_{i}\left(\sigma_{i}^{*}, s_{-i}\right)$. Therefore, by Definition 5 the strategy $\sigma^{*}$ is not an MBE to yield a contradiction.

Conversely, suppose $\sigma^{*}$ is a strategy such that if

$$
u_{i}\left(\sigma_{i}^{*}, s_{-i}\right)<\max _{s_{-i} \in S_{-i}} u_{i}\left(\sigma_{i}^{*}, s_{-i}\right),
$$

then $\sigma_{-i}^{*}\left(s_{-i}\right)=0$. Hence

$$
u_{i}\left(\sigma_{i}^{*}, \sigma_{-i}^{*}\right)=\max _{s_{-i} \in S_{-i}} u_{i}\left(\sigma_{i}^{*}, s_{-i}\right), \forall i \in I .
$$

Thus $\sigma^{*}$ is an MBE by Definition 5 to complete the proof.

We now use a counterexample to prove that an MBE may not exist in $n$-person extensive form games with $n \geq 3$.

Theorem 4. An MBE may not exist when $n \geq 3$.

Proof. The proof of this theorem is by a counterexample. Consider the following example of Figure 2. We claim that there is not an MBE for this game. Suppose there exists an MBE $\sigma^{*}$ for the game. Let $\sigma_{1}^{*}$ be the strategy of player 1. Note that from Figure 2

$$
\max _{s_{-1} \in S_{-1}} u_{1}\left(\sigma_{1}^{*}, s_{-1}\right)=1 \text {. }
$$

Moreover, $\sigma^{*}$ is an MBE. Hence players 2 and 3 choose with positive probabilities their pure strategies that give player 2 a payoff 1 . Hence player 2 would only choose strategy $s_{2}, t_{2}$. However, whenever player 2 wants to maximize player 1 's payoff there exists a pure strategy for player 3 such that for some pure strategy for player 1 in $\operatorname{supp}\left(\sigma_{1}^{*}\right)$

$$
\max _{s_{-2} \in S_{-2}} u_{2}\left(\sigma_{2}^{*}, s_{-2}\right)=1
$$

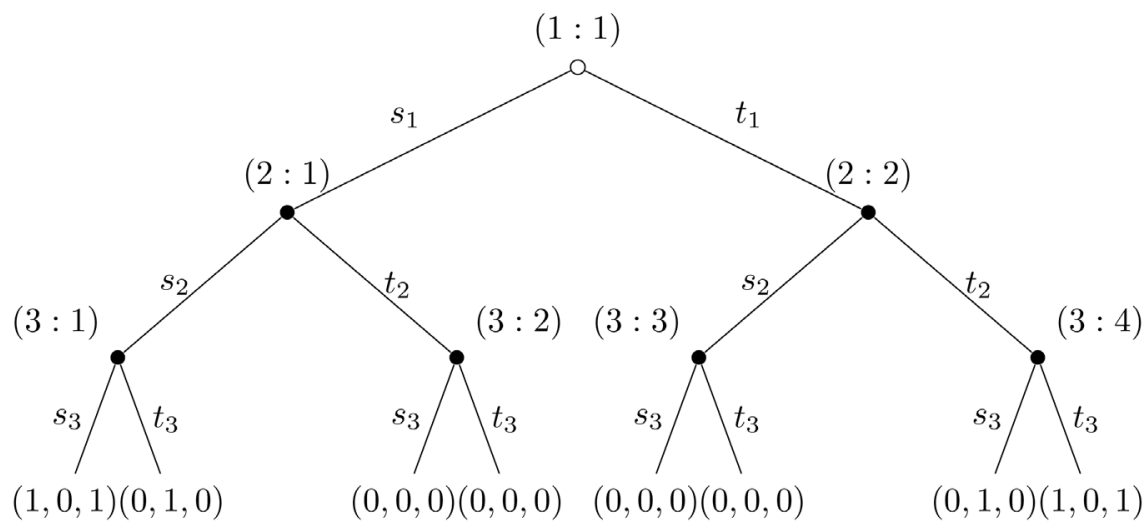

Figure 2. Three-person game with no MBE. 
Any strategy chosen by player 3 can only maximize either player 1's or player 2's expected payoff, but not both. Hence $\sigma^{*}$ cannot be an MBE by Lemma 3 to yield a contradiction.

\section{Examples}

In this section we give two examples. In the first example we consider a 3-person game with imperfect information. We show that the game does not have an MBE. If we consider the same game with perfect information, then it has an MBE. However, the game does not have an SPMBE even with perfect information.

\section{Example 1:}

We now show an example of an imperfect information game. Consider the 3-person game shown in Figure 3. The game shown in Table 1 is the normal form representation for the game in Figure 3. However, it was proven in [3] that an MBE does not exist for this game. We now consider the same game but with perfect information as shown in Figure 4. An interesting result is that the game has multiple MBEs in the case of perfect information.

The strategies for player 1 are simply $s_{1}$ and $t_{1}$. However, player 2 has 4 pure strategies and player 3 has 16 pure strategies, as shown in Table 2 and Table 3 respectively.

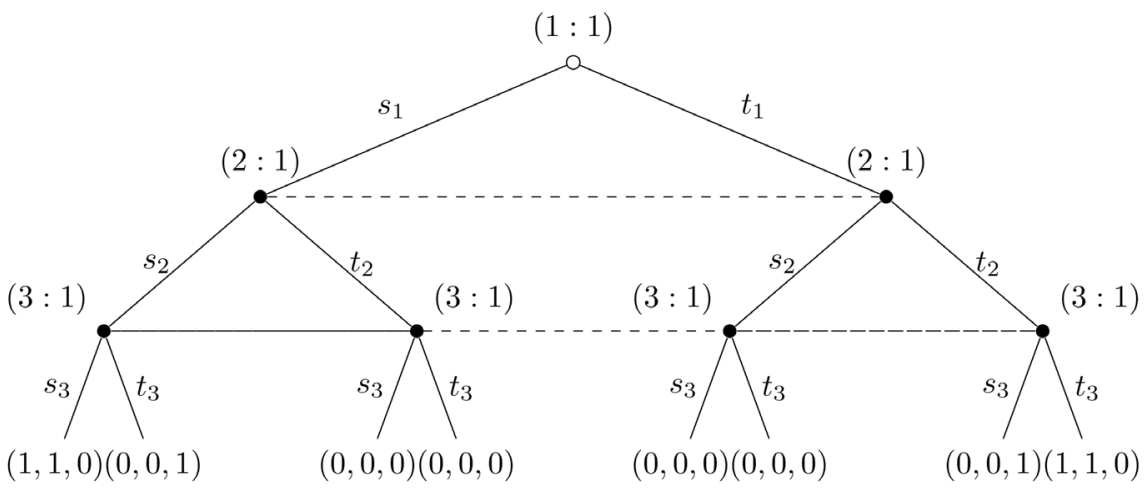

Figure 3. Three-person game with imperfect information.

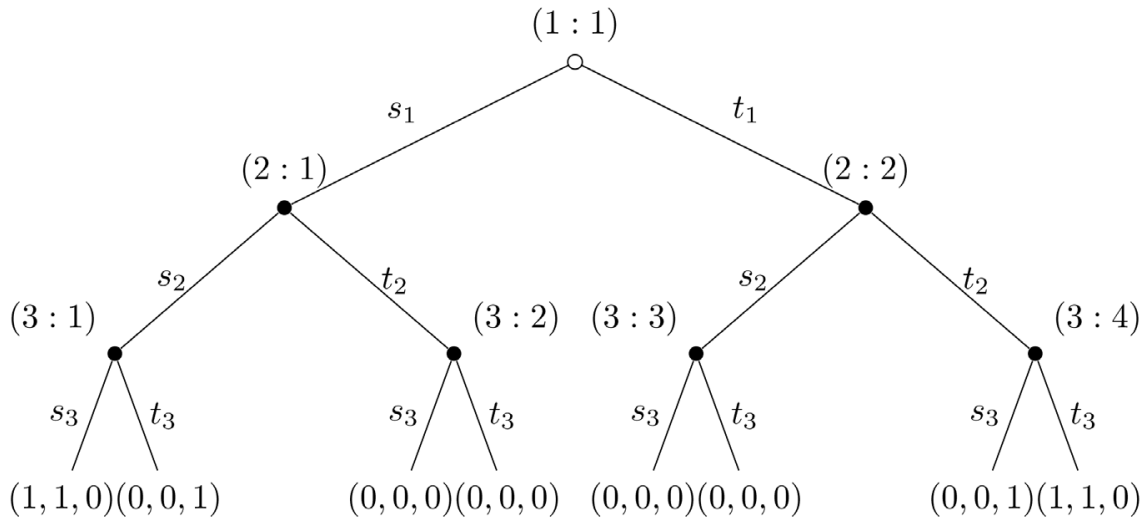

Figure 4. Three-person game with perfect information. 
Table 1. Normal form representation.

\begin{tabular}{cccccc}
\hline$s_{3}$ & $s_{2}$ & $t_{2}$ & $t_{3}$ & $s_{2}$ & $t_{2}$ \\
\hline$s_{1}$ & $(1,1,0)$ & $(0,0,0)$ & $s_{1}$ & $(0,0,1)$ & $(0,0,0)$ \\
$t_{1}$ & $(0,0,0)$ & $(0,0,1)$ & $t_{1}$ & $(0,0,0)$ & $(1,1,0)$ \\
\hline
\end{tabular}

Table 2. Player 2's strategies.

\begin{tabular}{ccc}
\hline Player 2's pure strategies & \multicolumn{1}{c}{$s_{1}$} \\
\hline Strategy 1 & If player 1 chooses $s_{1}$, then $s_{2}$. If player 1 chooses $t_{1}$, then $s_{2}$. \\
Strategy 2 & If player 1 chooses $s_{1}$, then $s_{2}$. If player 1 chooses $t_{1}$, then $t_{2}$. \\
Strategy 3 & If player 1 chooses $s_{1}$, then $t_{2}$. If player 1 chooses $t_{1}$, then $s_{2}$. \\
Strategy 4 & If player 1 chooses $s_{1}$, then $t_{2}$. If player 1 chooses $t_{1}$, then $t_{2}$. \\
\hline
\end{tabular}

Table 3. Player 3's strategies.

\begin{tabular}{ccccc}
\hline Player 3's pure strategies & $s_{1}, s_{2}$ & $s_{1}, t_{2}$ & $t_{1}, s_{2}$ & $t_{1}, t_{2}$ \\
\hline Strategy 1 & $s_{3}$ & $s_{3}$ & $s_{3}$ & $s_{3}$ \\
Strategy 2 & $s_{3}$ & $s_{3}$ & $s_{3}$ & $t_{3}$ \\
Strategy 3 & $s_{3}$ & $s_{3}$ & $t_{3}$ & $s_{3}$ \\
Strategy 4 & $s_{3}$ & $t_{3}$ & $s_{3}$ & $s_{3}$ \\
Strategy 5 & $s_{3}$ & $s_{3}$ & $t_{3}$ & $t_{3}$ \\
Strategy 6 & $s_{3}$ & $t_{3}$ & $t_{3}$ & $s_{3}$ \\
Strategy 7 & $s_{3}$ & $t_{3}$ & $s_{3}$ & $t_{3}$ \\
Strategy 8 & $s_{3}$ & $t_{3}$ & $t_{3}$ & $t_{3}$ \\
Strategy 9 & $t_{3}$ & $t_{3}$ & $s_{3}$ & $s_{3}$ \\
Strategy 10 & $t_{3}$ & $t_{3}$ & $s_{3}$ & $s_{3}$ \\
Strategy 11 & $t_{3}$ & $t_{3}$ & $s_{3}$ & $s_{3}$ \\
Strategy 12 & $t_{3}$ & $s_{3}$ & $s_{3}$ & $s_{3}$ \\
Strategy 13 & & $s_{3}$ & $s_{3}$ \\
\hline
\end{tabular}


For this game, player 3 has 16 different strategies as shown in Table 3. For example strategy 1 means that if player 1 chooses $S_{1}$, and player 2 chooses $S_{2}$, then player 3 chooses $s_{3}$. If player 1 chooses $s_{1}$, and player 2 chooses $t_{2}$, then player 3 chooses $s_{3}$. If player 1 chooses $t_{1}$, and player 2 chooses $s_{2}$, then player 3 chooses $s_{3}$. If player 1 chooses $t_{1}$, and player 2 chooses $t_{2}$, then player 3 chooses $S_{3}$.

One BE for this game is that player 1 chooses $s_{1}$, player 2 chooses $s_{2}, s_{2}$, and player 3 chooses strategy $s_{3}, s_{3}, t_{3}, t_{3}$. Note that for this BE, player 3 gets a payoff 0 . However, players 1 and 2 cannot increase player 3 's payoff regardless of their strategies. Moreover, they want to maximize the expected payoff for each other. Hence the strategy is an MBE.

Note that even with perfect information, the game does not have an SPMBE. Using backward induction, regardless of whether player 3 chooses $s_{3}$ or $t_{3}$, then player 1 alone can increase player 3's expected payoff. However that would result in reducing player 2's expected payoff. A symmetric result holds for player 1 if player 2 increases player 3's payoff. Hence the game cannot have an SPMBE. The next remark follows immediately.

Remark. An MBE for the game $\mathrm{G}$ is not necessarily an SPMBE.

\section{Example 2:}

We now give an example of a 2-person Bayesian game. Bayesian games with different types have been considered in literature; e.g., see [5]. In this example, we consider a 2-person game in extensive form. Each player has two strategies cooperate (C) and defect (D). We assume that there is a probability distribution over the types of player 1 . The first type is an altruistic type. This type wants to maximize player 2's expected payoff. The second type chooses the strategy Tit-for-Tat of [6]. The second type will cooperate with player 2 only if player 2 chooses to cooperate with player 1 . The third type is selfish and wants to maximize his own expected payoff. In this example, let the probability of each type be $P$ [Type 1$]=p_{1}$, $P\left[\right.$ Type 2] $=p_{2}$ and $P[$ Type 3$]=p_{3}$. The game is an imperfect information game. We assume that the game is repeated and not a one-stage game. At each stage, player 1 can be from any type and player 2 only knows the probability distribution over the types. Player 2 next chooses his action. Then player 1 chooses his action without knowing what action player 2 chose. The payoffs for each are shown in Figure 5.

The normal form representation of the game in Figure 5 is shown in Table 4.

Note that for $p_{1} \geq 0.9$ an NE for the game would be $(C, D)$. Hence player 2 would always defect. In the case that $p_{3} \geq 0.9$, an NE would be $(D, D)$. However, when $p_{2} \geq 0.9$, then an NE for the game is $(C, C)$. In all three cases, player 2 is selfish and concerned with his own payoff. Hence he would rather defect unless there is a high probability for the Tit-for-Tat type where player 2 can maximize his expected payoff by cooperating if the game is repeated.

\section{Conclusion}

The MBE is a solution concept in game theory that represents mutual cooperation 


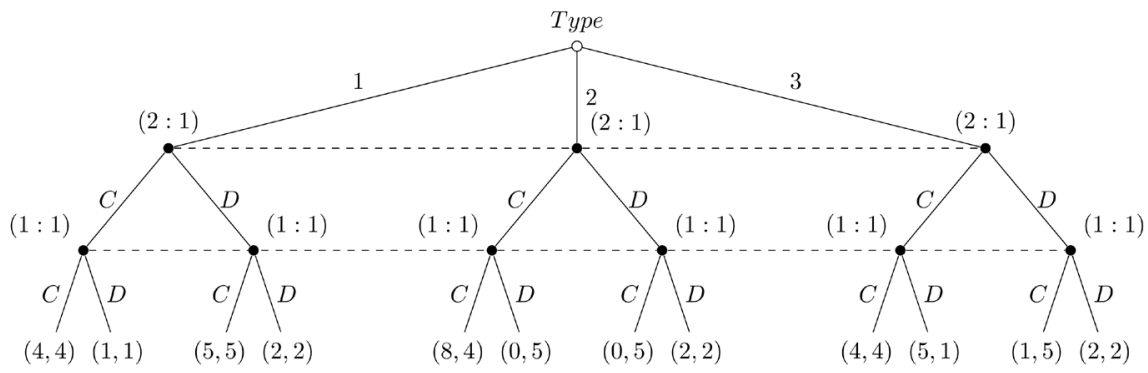

Figure 5. Two-person Bayesian game in extensive form.

Table 4. Two-person Bayesian game in normal form.

\begin{tabular}{ccc}
\hline & $s_{2}$ & $t_{2}$ \\
\hline$s_{1}$ & $\left(4 \times p_{1}+8 \times p_{2}+4 \times p_{3}, 4\right)$ & $\left(5 \times p_{1}+0 \times p_{2}+1 \times p_{3}, 5\right)$ \\
$t_{1}$ & $\left(1 \times p_{1}+0 \times p_{2}+5 \times p_{3}, 1\right)$ & $(2,2)$ \\
\hline
\end{tabular}

among players and extends the $\mathrm{BE}$ to mixed strategies. In this paper, we extended extensive form games to include players acting altruistically. In particular, we applied the concept of an MBE to finite $n$-person games in extensive form. We showed how an MBE always exists for 2-person games. However, we showed that an MBE may not exist in an $n$-person extensive form games with $n \geq 3$. We extended the definition of the subgame perfect equilibrium to include the case of the MBE. Moreover we proved that an SPMBE may not exist for $n \geq 3$.

\section{Acknowledgements}

This research is funded by the U.S. Department of Education GAANN Fellowship, reward no. P200A130164.

\section{References}

[1] Berge, C. (1957) Theorie generale des jeux an personnes. Gauthier-Villars, Paris.

[2] Zhukovskiy, V. (1985) Some Problems of Nonantagonistic Differential Games. In: Kenderov, P., Ed., Matematiceskie Metody v Issledovanii Operacij, Bulgarian Academy of Sciences, Sofia, 103-195.

[3] Corley, H.W. (2015) A Mixed Cooperative Dual to the Nash Equilibrium. Game Theory, 2015, 1-7. https://doi.org/10.1155/2015/647246

[4] Osborne, M.J. and Rubinstein, A. (1994) A Course in Game Theory. MIT Press, Boston.

[5] Barron, E.N. (2013) Game Theory: An Introduction (Vol. 2). John Wiley \& Sons, Hoboken. https://doi.org/10.1002/9781118547168

[6] Axelrod, R. and Axelrod, R.M. (1984) The Evolution of Cooperation (Vol. 5145). Basic Books, New York. 